\title{
Synthetic Biology Construct of Ebola Virus in Bacteria Surrogate Is Stable and Safe for Rapid Detection Studies in a BSL-2 Laboratory Setting
}

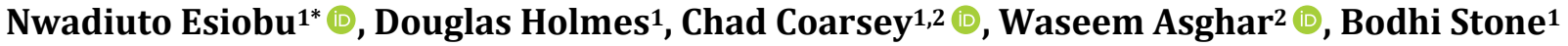 \\ ${ }^{1}$ Microbial Biotech Laboratory, Biological Sciences Department, Florida Atlantic University, Boca Raton Florida, USA \\ ${ }^{2}$ Department of Electrical Engineering and Computer Science, Florida Atlantic University, Boca Raton, Florida, USA \\ Email: *Nesiobu@fau.edu
}

How to cite this paper: Esiobu, N., Holmes, D., Coarsey, C., Asghar, W. and Stone, B. (2022) Synthetic Biology Construct of Ebola Virus in Bacteria Surrogate Is Stable and Safe for Rapid Detection Studies in a BSL-2 Laboratory Setting. Advances in Microbiology, 12, 25-41.

https://doi.org/10.4236/aim.2022.121003

Received: November 24, 2021

Accepted: January 25, 2022

Published: January 28, 2022

Copyright $\odot 2022$ by author(s) and Scientific Research Publishing Inc. This work is licensed under the Creative Commons Attribution International License (CC BY 4.0).

http://creativecommons.org/licenses/by/4.0/

(c) (i) Open Access

\begin{abstract}
Rapid detection of virulent pathogens during an outbreak is critical for public health advisories and control of the disease in a population. While many molecular techniques for point of care and clinical diagnosis abound, the US experience with the COVID-19 testing in the early stages of the pandemic underscores the critical importance of determining the appropriate target gene(s) with in-built controls that reliably detect pathogens with high sensitivity and specificity. Assays and research for diagnostics and therapy could be slowed during an epidemic because access to the required BSL- 3 and BSL-4 laboratories are limited. So, during the 2014 West Africa Ebola outbreak, we tested the hypothesis that using synthetic cDNA of Ebolavirus in a bacteria surrogate (fit for all lab settings), would remain unmutated and safe after several generations, serving as an effective positive control in research settings, self test and point-of-care detection platforms. Primers were designed for the detection and quantification of the nucleoprotein (NP) gene of the 2014 Makona Ebola strain (KR781608.1, 733 - 1332 bp). To test the stability of artificially inserted translation arrest in the Orf of the model gene, it was edited to include three STOP codons in the RNA transcript using SNAP GENE. The segment was then spliced into a high copy number plasmid, cloned into One Shot ${ }^{\mathrm{TM}}$ TOP10 Escherichia coli (Invitrogen), and tested for stability and safety by periodic subculture, extraction and sequencing. Unlike COVID-19, rapid detection of blood-borne etiologies like Ebola requires optimized protocols for blood matrix. Using real-time PCR and newly designed primer pairs, the EBOV surrogate was detected and enumerated in human blood and regular broth and buffers. Based on aligned sequence analysis, the EBOV synthetic NP gene was stable ( $>99.9999 \%$ similarity coefficient) for at least 3 months. Detection sensitivity in broth and blood was at least 100 cells $/ \mathrm{ml}$ or about 5.8
\end{abstract}


$\times 10^{3}$ to $7.3 \times 10^{3}$ virion equivalents per $\mathrm{ml}$. While the developments of transcription-and-replication-competent virus like particles (trVLP) have made it possible to study the infection and replication cycles of virulent pathogens in BSL-2 laboratories, the simplicity of our model and the reproducibility of detection and enumeration show the utility of synthetic bio-components as positive controls for point of care diagnostic tools. The inserted stop codons remained intact after many generations, suggesting that expressed virulent proteins can be easily silenced in synthetic biology models for research in BSL-1 and 2 and a wide range of pathogens. Synthetic bio-components can thereby aid further research by reducing costs and improving safety for workers and stakeholders.

\section{Keywords}

Ebola Virus, Synthetic Biology, Biosafety, Point-of-Care, Rapid Detection, Plasmid Vector

\section{Introduction}

Ebola virus (EBOV) is a single-stranded, negative-sense RNA virus. Electron micrographs show virions to be $80 \mathrm{~nm}$ thick and 800 - $1400 \mathrm{~nm}$ in length, dimensions that characterize the family filoviridae [1]. The EBOV genome contains approximately 19 kilobases and 7 genes: glycoprotein (GP), nucleoprotein (NP), RNA dependent RNA polymerase (RdRP), VP24, VP30, VP35, and VP40 [2]. Human infection with EBOV causes Ebola Virus Disease (EVD), hemorrhagic fever with a case mortality rate up to $90 \%$ [3]. EBOV virions consist of a helical nucleocapsid and cylindrical lipid envelope, and are spread to new hosts via contact with contaminated blood or body fluids from an infected individual. Outside of a host, the virions retain viability on surfaces for several days, and in fluids for up to several weeks [4]. Outbreaks of EVD have been extensively documented and the epidemiology of the disease is well known [4]. During an outbreak, healthcare and laboratory workers are at high risk of infection in regions with limited resources for transmission prevention and mitigation [5]. These features make a level 4 biosafety rating (BSL-4) a requirement for EBOV research [6], a fact that limits the number of researchers studying the virus.

Recently, the global SARS-COV-2 pandemic has highlighted the importance of early detection to limit transmission of the virus [7]. Detection and diagnosis of EBOV infection are not possible before the onset of symptoms, which can be non-specific in early stages of EVD [8]. Approved EBOV detection techniques fall under three categories: nucleic acid amplification, immunoassay, and virus isolation [8]. Nucleic acid amplification with reverse transcriptase PCR (RT-PCR) affords the highest sensitivity in the shortest amount of time, able to generate results for a single virion per reaction volume [9]. Loop-mediated isothermal amplification (LAMP) is another detection method that generates rapid results 
with minimal technology [10]. LAMP has not yet been used to detect genuine EBOV virions but has potential for point-of-care detection in EBOV diagnostics [11].

Although new achievements in EBOV research include the first vaccine against Zaire ebolavirus, Ervebo [12], and the monoclonal antibody treatments, Inmazeb and Ebanga [13], they do not obviate the desirability of model systems to study without risk of infection or illness, and without need of a BSL-4 laboratory. For the study of the EBOV lifecycle, the innovative tetracistronic minigenome system uses a transcription and replication-competent virus-like-particle (trVLP). The trVLP is a plasmid with a cDNA copy of the EBOV genome and deletions of genes encoding the proteins VP30, VP35, NP, and L. The remaining genes make up the tetracistron: VP40, VP24, $\mathrm{GP}_{1,2}$, and a reporter. Infection, maturation, and budding can be monitored within host cells that are transfected with expression plasmids for the deleted genes, RdRP, and the tetracistronic minigenome [14]. Subsequent infection and budding will continue only so long as target cells are supplied with the expression plasmids, which allow the EBOV tetracistronic minigenome to be studied in a BSL-2 laboratory.

The use of various forms of minigenome, including the trVLP (reverse genetics systems for filoviruses and other negative-stranded hemorrhagic fever viruses) is an important albeit limited tool used to study the life cycle of Ebola viruses in plasmid-based expression viral proteins under BSL-2 settings [15]. Subsequent advances resulted in the development of multi-cistronic minigenome model systems that directly express a reporter and viral genes rather than from an expression plasmid, allowing the study of most aspects of the EBOV life cycle over the course of multiple infection cycles in susceptible host cells [14]. Nonetheless, using synthetic biology to create rapid and safe protocols for the development of point-of-care detection methods and positive controls in a BSL-2 laboratory in a cost-efficient manner, remain an essential need. During the 2014-2016 Ebola outbreak there were no EBOV vaccines and no FDA-approved treatments for EVD [16]. Rapid virus detection in blood and body fluids matrices at airports, for instance, was a priority. The acute need for a synthetic gene surrogate that is a stable mono-cistronic subgenome for point-of-care detection research in BSL-2 laboratories inspired the activities of the studies reported in this manuscript. Synthetic biology has found many practical applications in life science technologies, including in silico directed evolution of novel protein functions [17]. The genetic material of any organism (or replicating virus) can be modified to eliminate pathogenicity while still retaining identity and integrity. Targeted molecular processes for these modifications are well known-demobilization of translation, modifying open reading frames into noncoding sequences, and recombinants expressing firefly luciferase genes as reporters. Such geneediting is sometimes required for regulatory approvals to work with a potentially pathogenic synthetic gene in a BSL-2 surrogate. A parallel concern is the selection of a suitable, non-pathogenic host for the synthetic gene construct when 
cloning, and or protein expression are desired. In this study we used a sub-monocistronic cDNA designed from the EBOV NP gene to simulate viral titer and detection, without risk to researchers and the public. To test the feasibility of the synthetic EBOV surrogate gene model and point-of-care detection methods, we hypothesized that if a synthetic EBOV gene segment were inserted into a plasmid, and then cloned in suitable bacteria host cell, that the gene sequence will remain unmutated and would be detectable by reporter probes and $\mathrm{QPCR}$ at similar rates to the same surrogates in blood matrix.

\section{Materials and Methods}

\subsection{Gene Selection and Bioinformatics}

The EBOV NP protein is integral to the generation of new virions and is relatively conserved [18]. Its purpose in this model is to demonstrate that the plasmid surrogate will maintain sequence integrity over many generations with minimal mutation. The gene was identified in NCBI RefSeq and run through a BLAST protocol to determine any areas of particularly strong conservation [19]. In order to gain CDC approval for work with the NP gene in BSL-2, a section of approximately $600 \mathrm{bp}$ was selected and modified by inserting stop codons at the 5 ' end, at the 3' end, and after every methionine codon, making translation unlikely. The segment was then flanked by restriction sites found in the pUC19 Multiple Cloning Site (MCS) region. SnapGene 4.0 nucleic acid visualization software was used to perform these modifications. The sequence was synthesized de novo and ligated into a plasmid by Eurofins Genomics. For the nucleocapsid gene, $600 \mathrm{bp}$ were found and flanked by SmaI in the 5' direction and EcoRI in the 3 ' direction, and this fragment was then inserted into pEX-A2, a streamlined high copy number plasmid featuring a convenient MCS, ampicillin resistance, and an origin of replication (Ori) identical to the pUC series.

\subsection{Transformation of E. Coli}

Invitrogen One Shot ${ }^{\mathrm{TM}}$ TOP10 Chemically Competent E. coli was removed from $-80^{\circ} \mathrm{C}$ and placed on ice for 20 minutes. $12.5 \mathrm{ng}$ of pEX-A2 with Ebola insert $(12.5 \mathrm{ng} / \mu \mathrm{l})$ or unmodified pUC19 $(5 \mathrm{ng} / \mu \mathrm{l})$ was added to $50 \mu \mathrm{l}$ of $E$. coli and allowed to sit for 25 minutes. After that time, the cells were heat-shocked at $42^{\circ} \mathrm{C}$ for 45 seconds and then returned to ice for 2 minutes. $450 \mu$ of Luria Broth (LB) were then added to the cells, and $100 \mu \mathrm{l}$ each of these cultures were plated on 3 LB plates supplemented with $100 \mu \mathrm{g} / \mathrm{ml}$ ampicillin and $4 \mu \mathrm{l} / \mathrm{ml} \mathrm{x}$-gal/IPTG solution. These plates were then incubated overnight at $37^{\circ} \mathrm{C}$.

\subsection{Purification of Plasmid and Genomic DNA}

Genomic DNA was purified by use of the Qiagen DNeasy Blood \& Tissue Kit, and plasmid DNA was purified using the QIAprep Spin Miniprep Kit as directed for bacterial cultures. The nucleic acid concentration was determined by spectrophotometry with a Nanodrop 2000 c Spectrophotometer. 


\subsection{PCR Confirmation of Plasmid Surrogate}

Polymerase Chain Reaction (PCR) amplification was performed to determine that the target insert was present. For both inserts, primer sequences were created using SnapGene software, and PCR was performed to the specifications of those primers (Table 1 ).

$12.5 \mu \mathrm{l}$ of $2 \times$ Master mix was combined with $1 \mu$ forward primer, $1 \mu \mathrm{l}$ reverse primer, $1 \mu \mathrm{l}$ Bovine Serum Albumin (BSA), and $1 \mu \mathrm{l}$ of sample from each purified plasmid sample. This procedure was repeated using $1 \mu \mathrm{l}$ of a lysed cell extract obtained from $1 \mathrm{ml}$ of cell culture. The culture was centrifuged at $6000 \mathrm{~g}$ for 5 minutes, and $50 \mu \mathrm{l}$ of Tris-EDTA (TE) buffer with $0.1 \mathrm{mM}$ EDTA was added. The sample was then heated at $100^{\circ} \mathrm{C}$ for 5 minutes and vortexed for 30 seconds to suspend the DNA in solution. PCR results were then run on a $1 \%$ agarose TAE gel.

\subsection{Surrogate Stability Testing}

The stability of the surrogate was determined by isolating 100 colonies and transferring them to two plates, one containing ampicillin, one without. Colonies from the plate without ampicillin were transferred as described above, repeated for ten sets of plates (Figure 1). The ampicillin plate shows which colonies maintain the plasmid and which-if any-lose the plasmid over multiple rounds of replication.

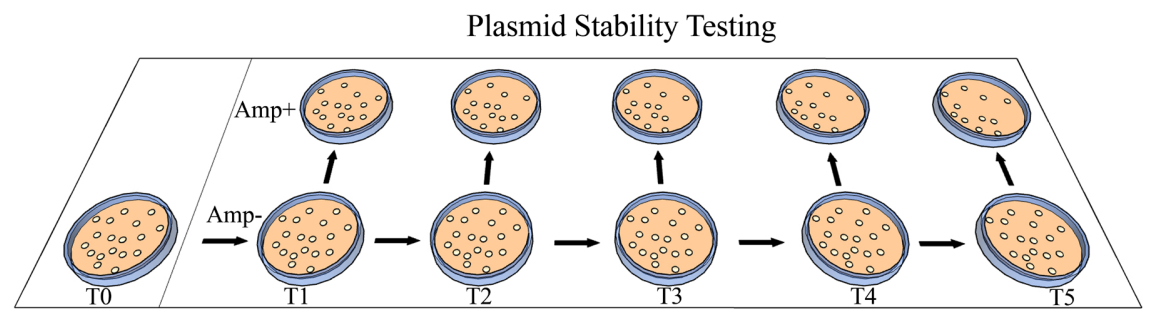

Figure 1. Serial transfer plates for plasmid stability Testing: plates in the bottom row lack ampicillin (Amp-), and therefore do not select for cells transformed with pEX-A2. Plates in the top row contain ampicillin (Amp+) and select for cells transformed with pEX-A2. Plasmid stability is tested by replica-plating colonies from Amp- to Amp+ plates. Only colonies that retain pEX-A2 will grow on the Amp+ plates. Note that only five sets of colony transfers are present in this figure, however, 10 sets of colony transfers were used.

Table 1. Primers designed in this study and PCR protocols. Note that all primers were designed in SnapGene and were suitable for both PCR and qPCR.

\begin{tabular}{cc}
\hline NP Forward Primer & $5^{\prime}$-CTCCATCCTCTTGCAAGGAC-3' \\
NP Reverse Primer & $5^{\prime}$-GGTCAAGTTCACGAGACTCC-3' \\
PCR Protocol & $94^{\circ} \mathrm{C}, 2 \mathrm{~min} ;\left[94^{\circ} \mathrm{C}, 1 \mathrm{~min} ; 57^{\circ} \mathrm{C}, 1 \mathrm{~min} ; 72^{\circ} \mathrm{C}, 30 \mathrm{sec}\right] \times 30 ;$ \\
$72^{\circ} \mathrm{C}, 3 \mathrm{~min} ; 4^{\circ} \mathrm{C}$, hold \\
qPCR Protocol & $95^{\circ} \mathrm{C}, 15 \mathrm{~min} ;\left[94^{\circ} \mathrm{C}, 15 \mathrm{sec} ; 57^{\circ} \mathrm{C}, 1 \mathrm{~min}\right] \times 40 ; 4^{\circ} \mathrm{C}$, hold \\
\hline
\end{tabular}




\subsection{Plasmid Surrogate Sequencing}

Because the specific nucleotide sequence of the plasmid surrogate is integral to the model, the plasmid was extracted from the colonies and sequenced to determine the exact sequence of the recombinant region. The plasmid was extracted from colonies on both the initial transfer plate (plate T1 above) and the final transfer plate (T10) for both surrogates. The randomly selected colonies were grown in Luria Broth (Fisher Scientific) overnight and their plasmid was extracted. The samples were then prepared for sequencing at Eurofins Genomics, then aligned using the SnapGene software to determine any mismatched bases. The differences in sequence of the insert region were used to determine an approximate rate of mutation for the insert.

\subsection{Surrogate Detection}

While authenticating the plasmid surrogate is important for determining the usefulness of the model for future testing, it is also necessary to prove the surrogate can be detected using standard molecular detection methods. For this, qPCR will serve as a contemporary detection technique for the surrogates. To determine the concentration of plasmid in cell culture, qPCR was performed on the recombinant section of the plasmid. Plasmid purified from cell culture was amplified and compared to the calculated cell density per milliliter of the cultures to determine the average plasmid count per cell. qPCR for both surrogates was carried out with the same primers as PCR, but the cycle program was modified to fit the specifications of qPCR (Table 1). Absolute Blue qPCR mix (Thermofisher) was used for the amplification and was prepared according to the manufacturer's instructions.

$1 \mathrm{ml}$ of overnight culture was collected and diluted to an OD of $1.2500 \mu \mathrm{l}$ of diluted culture was then centrifuged at $6000 \mathrm{~g}$ for 5 minutes, decanted, and $50 \mu \mathrm{l}$ of TE buffer was added. The sample was then heated at $100^{\circ} \mathrm{C}$ for 5 minutes and vortexed for 30 seconds to suspend the DNA in solution. The sample was then serially diluted in TE buffer 10:1 to $<1$ cell/ $\mu \mathrm{l}$, and $1 \mu \mathrm{l}$ of each was used for surrogate preparation. Samples were prepared in triplicate. Standards were created using an untransformed plasmid of a known concentration.

\subsection{Cell Spectrophotometry and Colony Plate Count}

$1 \mathrm{ml}$ of surrogate culture was placed in a cuvette and run through a Nanodrop 2000 c spectrophotometer to determine the Optical Density (OD) of the bacteria at $600 \mathrm{~nm}$, adjusted so that the OD is below 1 in the measured sample. The number of cells was then estimated using the equation $\mathrm{OD}_{600}=1.5 \times 10^{8}$ cells $/ \mathrm{ml}$. The cultures were then serially diluted such that the final dilution had a cell concentration less than $10^{3}$ cells $/ \mathrm{ml} .100 \mu \mathrm{l}$ of these dilutions were plated on a LB/Amp plate and incubated at $37^{\circ} \mathrm{C}$ overnight. Viable cells were then determined from the number of colonies grown to give a more accurate estimate of cells. 


\subsection{Blood Simulation}

To effectively simulate detection of EBOV in blood, $1 \mathrm{ml}$ samples of whole blood were prepared for nucleic acid purification and PCR amplification. The blood was spiked with bacterial surrogate to yield known concentrations, $10^{6}, 10^{4}$, and $10^{2}$ cells $/ \mathrm{ml}$, spun for 2 minutes at $2000 \mathrm{~g}$, and the serum collected for nucleic acid purification. $200 \mu \mathrm{l}$ of serum was then purified using ZymoBIOMICS ${ }^{\mathrm{TM}}$ DNA Miniprep Kit protocol for blood and other protein-rich fluids. PCR was then performed as above, and the results were run on a $2 \%$ agarose gel as above.

\section{Results}

\subsection{Dry Lab/Bioinformatics}

Ebola virus genomes from the four most important EBOV outbreaks (1979, 1995, 2001, and 2014) were downloaded from NCBI RefSeq and aligned with the EBOV type strain in SnapGene. Areas with minimal base pair mismatch were identified visually and their sequence recorded. The highly conserved regions were then sized for optimal insertion into the target plasmids. Large, highly conserved regions were found in both the GP and NP genes (Figure 2(a)).

From the bioinformatics performed during this project, a 600 bp segment from the nucleocapsid gene (Figure 2(b)) was identified and used to construct the plasmid surrogate.

Results from transforming E. coli with the recombinant NP surrogate (Figure 3 ) indicate that the plasmid was taken up effectively. All of the transformed colonies contained the recombinant plasmid, and E. coli transformed with pUC19 showed the x-gal/IPTG solution was working and present.

\subsection{Authentication}

Electrophoresis of the NP PCR amplification was also consistent with expectations

(a)

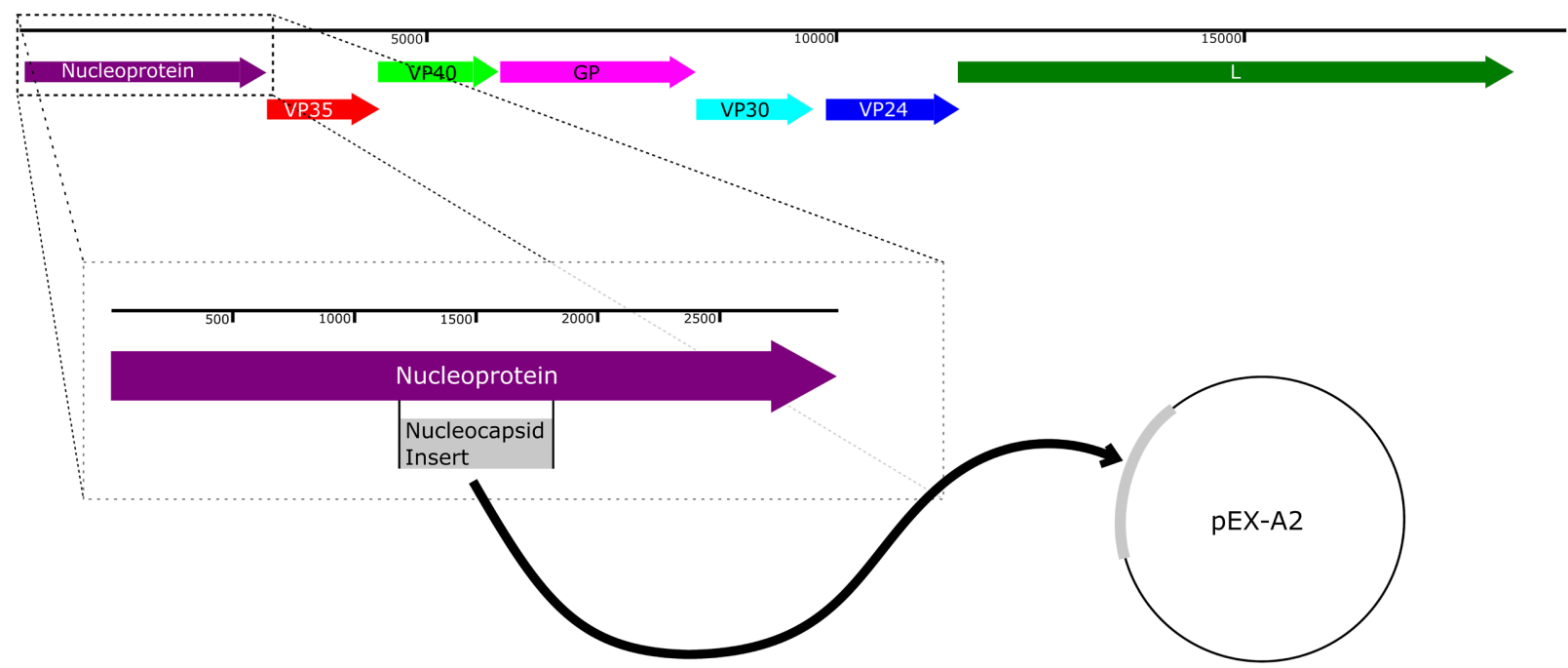

Figure 2. Annotation of EBOV: (a) location of the nucleoprotein (NP) gene; (b) region of the NP gene inserted into pEX-A2. 
pEX-A2 Vector Map

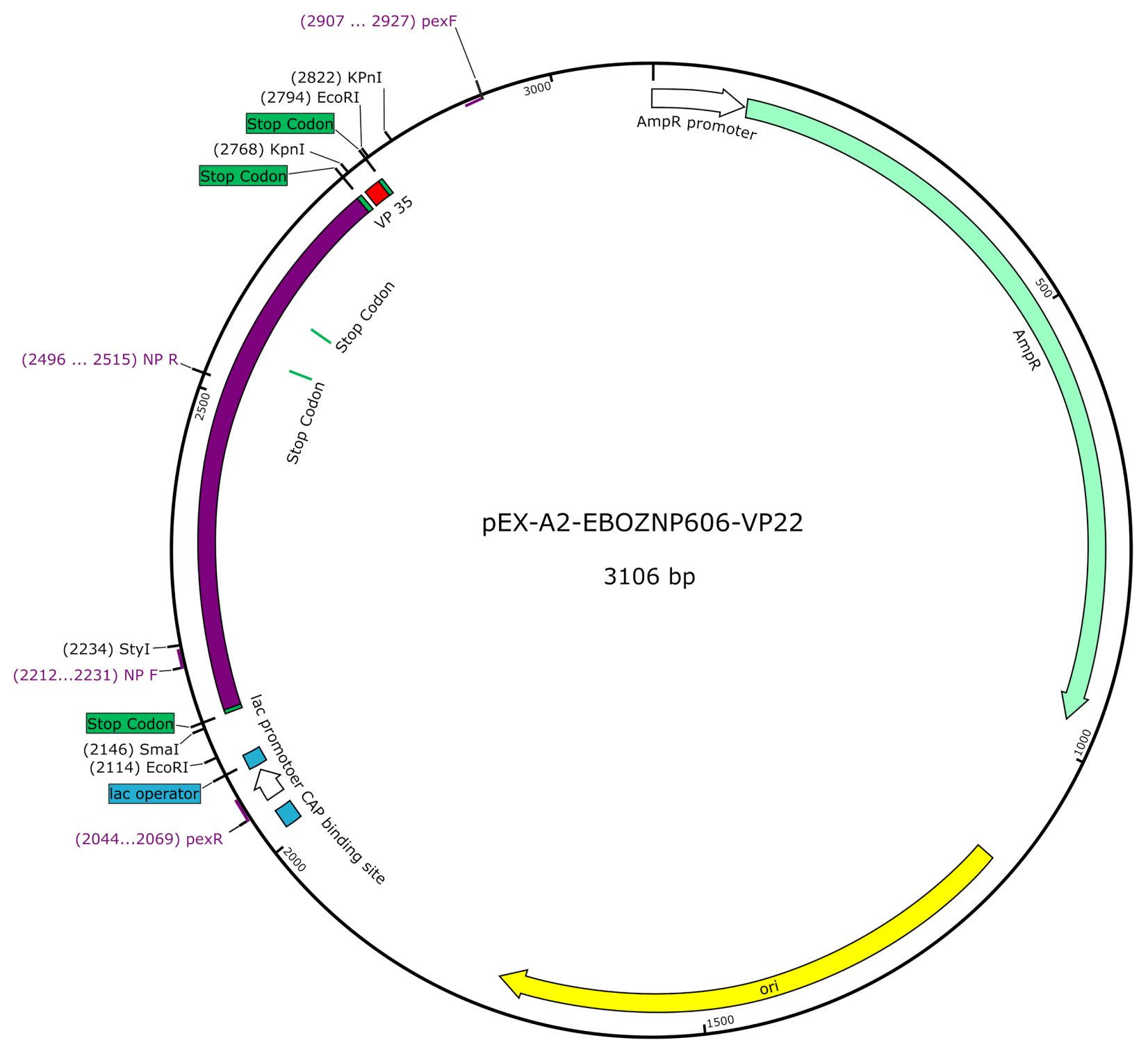

CCCGGGGGTAATTGATTGTCAAAACAGTACTTGATCATATCCTACAAAAGACAGAACGAGGAGTTCGTCTCCATCCTCTTGCAA GGACCGCCAAGGTAAAAAATGAGGTGAACTCCTTCAAGGCTGCACTCAGCTCCCTGGCCAAGCATGGAGAGTATGCTCCTTTC GCCCGACTITGAACCTTCTGGAGTAAATAATCTTGAGCATGGTCTITCCCTCAACTGTCGGCAATTGCACTCGGAGTCGCCA CAGCCCACGGGAGCACCCTCGCAGGAGTAAATGTTGGAGAACAGTATCAACAGCTCAGAGAGGCAGCCACTGAGGCTGAGA AGCAACTCCAACAATATGCGGACTCTCGTGAACTTGACCATCTTGGACTTGATGATCAGGAAAAGAAAATTCTTATGTAAAACT TCCATCAGAAAAAGAACGAAATCAGCTTCCAGCAAACAAACGCGATGTAAGTAACTCTAAGAAAAGAGCGCCTGGCCAAGCT GACAGAAGCTATCACTGCTGCATCACTGCCCAAAACAAGTGGACATTACGATGATGATGACGACATTCCCTITCCAGGACCCAT CAATGATGACGACAATCCTGGCCATCAAGATGATTAAGGTACCGATGAAGATTAAAACCTTCATTAAGAATTC

Figure 3. Recombinant NP plasmid surrogate. Insert sequences highlighted to correspond to plasmid diagrams (image from SnapGene ).

(Figure 4). A band of approximately $300 \mathrm{bp}$ was returned for all samples, and no amplification was seen in the negative controls. 


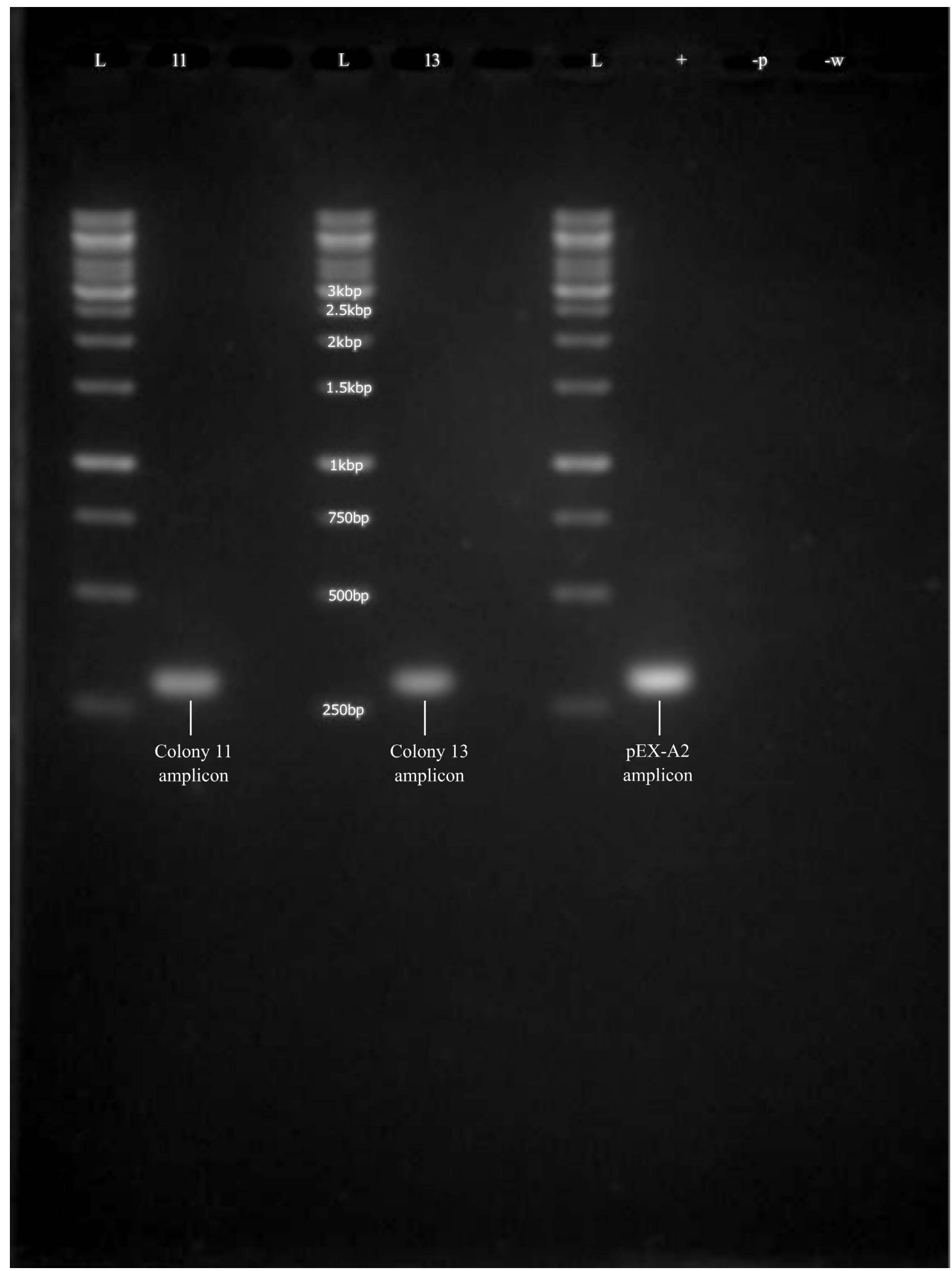

Figure 4. Gel electrophoresis of NP PCR. L = ladder (O’Generuler $1 \mathrm{~kb}$ plus), $11=$ transformed colony $11,13=$ transformed colony $13,+=$ pure recombinant plasmid, $-\mathrm{p}=\mathrm{pUC1}, \mathrm{s}, \mathrm{w}=$ water. 

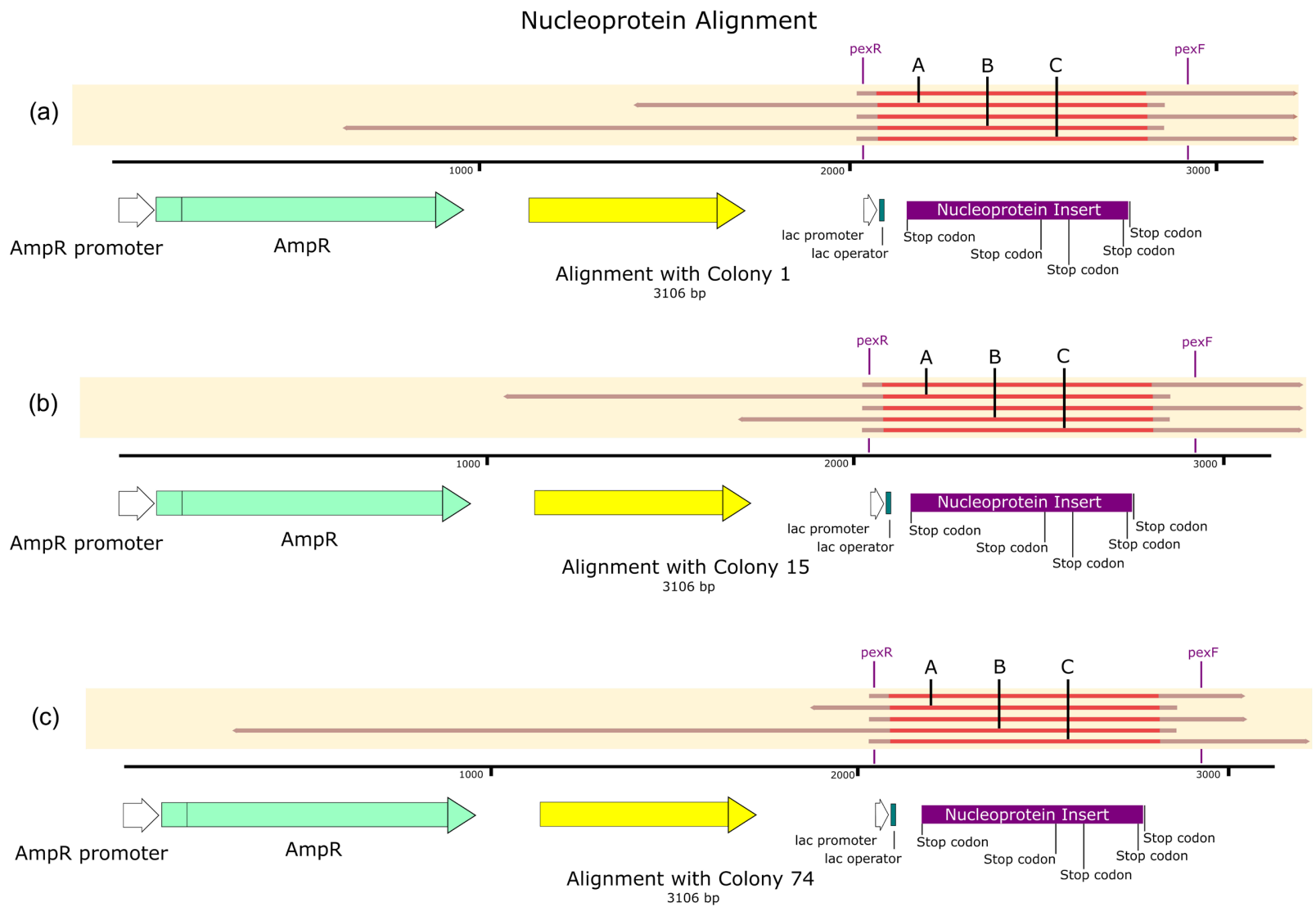

Figure 5. Sequence alignment of nucleoprotein colonies 1 (a), 35 (b), and 74 (c). (a) Corresponds to the colony sequenced from plate T0. (b) Corresponds to the colony sequenced from plate T10. (c) Corresponds to the original recombinant plasmid (image from SnapGene $)$. 


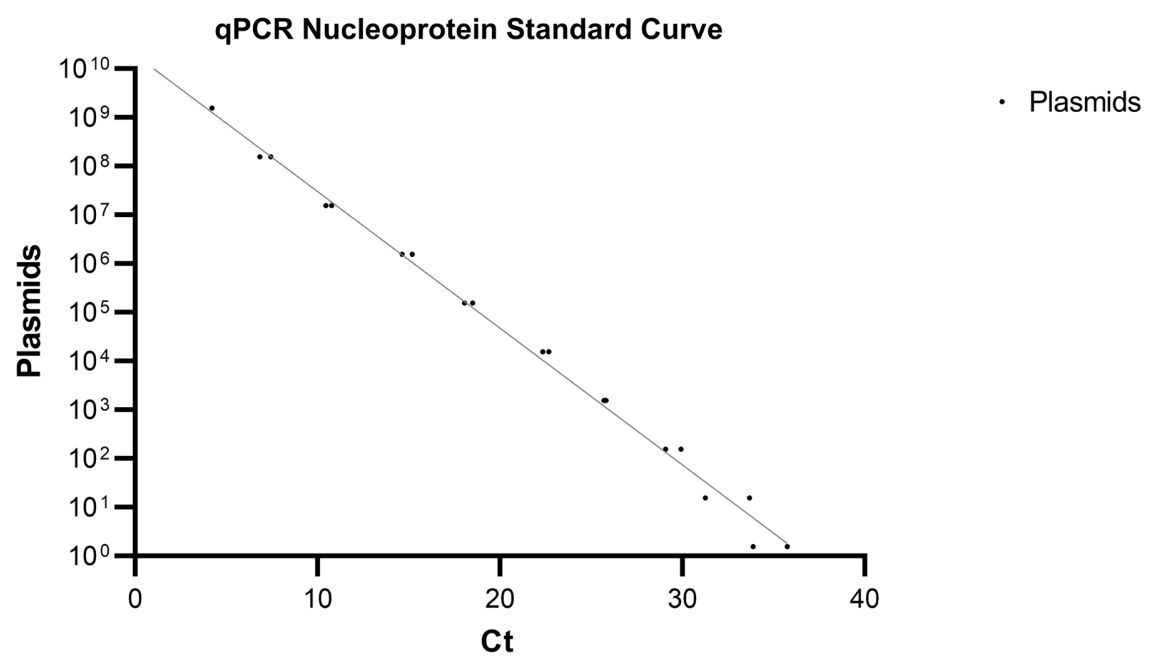

Figure 6. Standard curve for qPCR amplification of the nucleoprotein insert in pEX-A2. The curve was generated with known plasmid concentrations to correlate cycle threshold (Ct) values. Least squares regression analysis yielded a trendline with $\mathrm{R}^{2}=0.9936$. The trendline was then used to calculate unknown plasmid copy numbers from their respective Ct values.

qPCR results of the NP colony 11 (NP-11) surrogate indicated a concentration of between 59 and 73 plasmid equivalents per cell (Figure 7).

Attempts to detect the NP surrogate with nucleic acid extraction, purification, and PCR were successful. After interpolating plasmid concentration from the standard curve (Figure 6), qPCR Ct values indicated that plasmid equivalents per cell were approximately 10 fold greater in colony 11 compared to colony 13 . Above the threshold of $10^{2}$ cells the detection of plasmid equivalents increased steadily for both colonies. Finally, the nucleoprotein insert was detectable with the most dilute cell suspensions in the series.

Results from blood contamination simulation (Figure 8) show NP-11 surrogate cells were detected down to approximately $10^{2}$ cells $/ \mathrm{ml}$, or 20 cells in $200 \mu$ l. Based on analysis of plasmid equivalents per cell (Figure 7), this is equivalent to between $5.8 \times 10^{3}$ and $7.3 \times 10^{3}$ plasmid equivalents $/ \mathrm{ml}$ for the NP-11 surrogate.

\section{Discussion}

\subsection{Bioinformatics}

For these experiments the NP gene demonstrated the speed and flexibility of the surrogate creation process. The NP gene is one of the most common targets for detection, is relatively conserved, and its importance in EVD is considerable because of its role in virion assembly. Nucleic acid visualization software facilitated the disruption of all open reading frames, as required by the CDC for work in BSL-2. The sequence was truncated and modified with stop codons in silico to minimize the possibility of translation while disrupting the sequence as little as possible. Thus, the usefulness of the surrogate was maintained without compromising safety. 


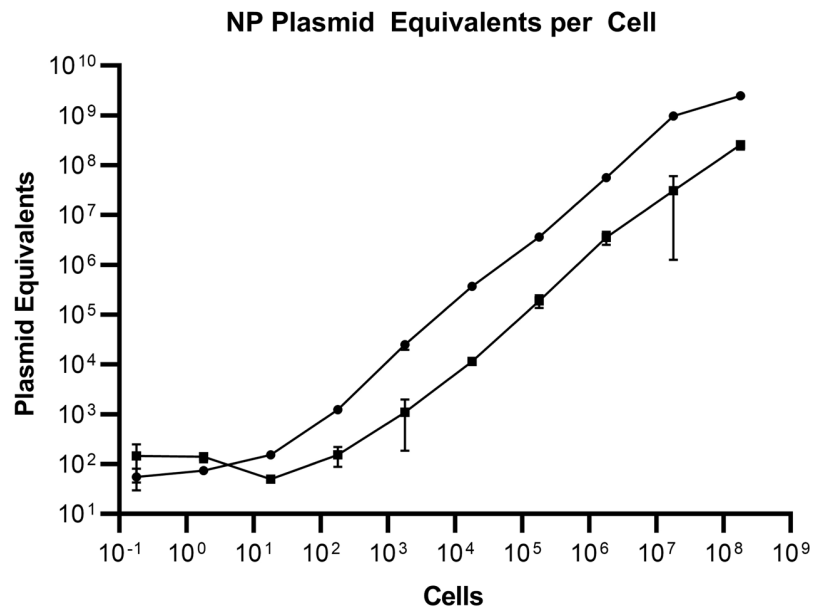

$\rightarrow$ NP colony 11
$\rightarrow \quad$ NP colony 13

Figure 7. To estimate nucleoprotein plasmid equivalents per cell, optical density from cultures 11 and 13 was used to measure cell density. Then, nucleic acid was extracted and purified, serially diluted tenfold, and the samples amplified with primers for the nucleoprotein insert in pEX-A2. Finally, the measured Ct values were interpolated with the standard curve in Figure 6 to calculate plasmid equivalents for each dilution. Error bars are not visible for data where they would be too small to see on the graph.

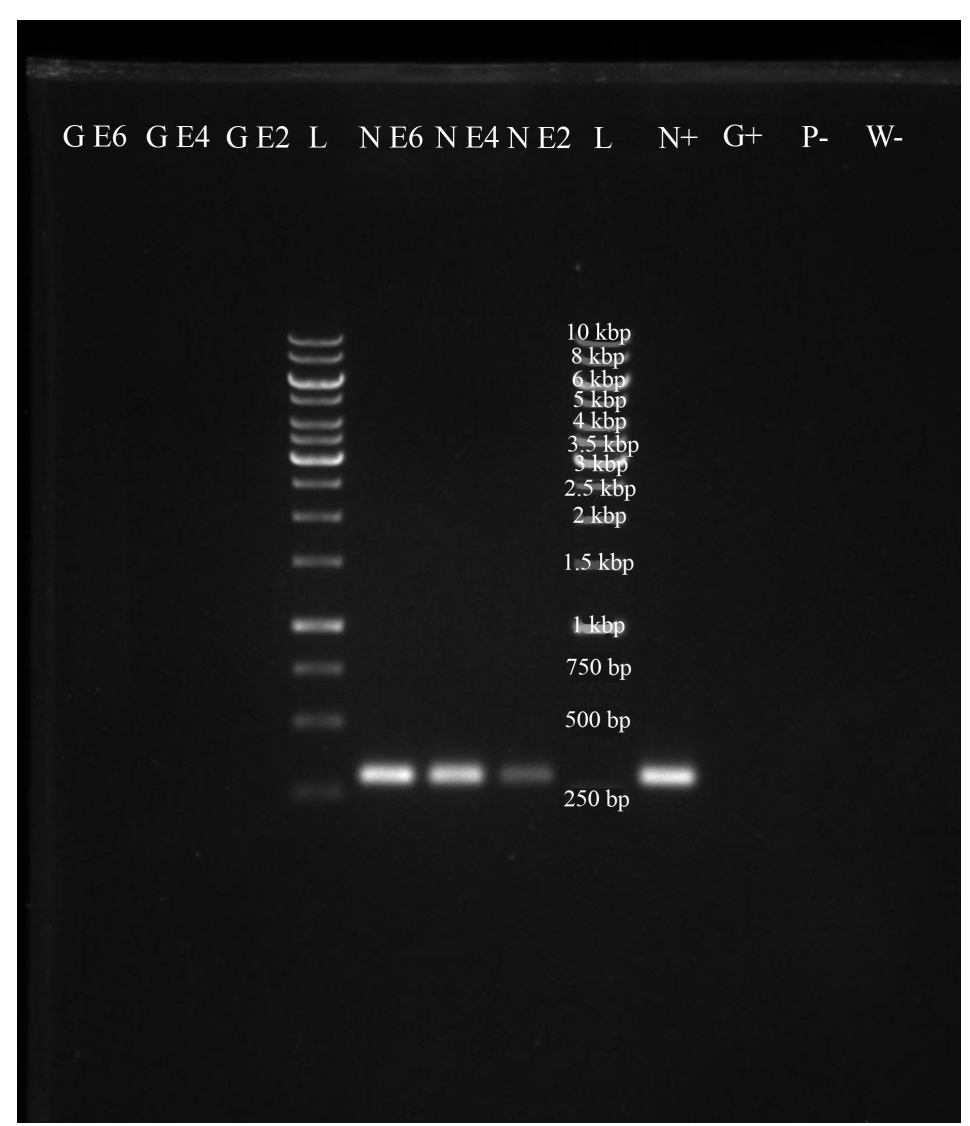

Figure 8. Gel electrophoresis of blood PCR targeting unsuccessful glycoprotein (GP) and nucleoprotein (NP) surrogates. L = ladder (O'Generuler $1 \mathrm{~kb}$ plus), GE6 $=10^{6} \mathrm{cells} / \mathrm{ml}$, GE4 $=10^{4}$ cells $/ \mathrm{ml}$, GE2 $=10^{2}$ cells $/ \mathrm{ml}, \mathrm{NE} 6=10^{6}$ cells $/ \mathrm{ml}, \mathrm{NE} 4=10^{4}$ cells $/ \mathrm{ml}, \mathrm{NE} 2=10^{2}$ cells $/ \mathrm{ml}, \mathrm{N}+=$ nucleoprotein insert positive control, $\mathrm{G}+=$ glycoprotein positive control, $\mathrm{P}-=$ pUC19 negative control, $\mathrm{W}-=$ Water. 


\subsection{Surrogate Construction}

The gene fragment was synthesized de novo with inserted stop codons to prevent translation and inserted into pEX-A2, a high copy number plasmid that encodes ampicillin resistance. This was then inserted into our selected $E$. coli strain, which was chosen for its low rate of mutation and chemically induced competence. These features made the transformation easy and relatively inexpensive, requiring no specialized equipment. Furthermore, E. coli is a relatively robust organism, and can grow on a number of different media, making it easy to use in most molecular biology laboratories. This is in contrast with EBOV, which requires very stringent and expensive containment procedures, and can only be used in the most well-equipped labs. The limitation of this technique is that the maximum size of the insert is limited, and while an insert could be created approximating a single $2 \mathrm{kbp}$ gene, it would be difficult to construct a surrogate for a larger gene. That said, the use of a cosmid or other larger-scale genetic construct would allow for a larger surrogate to be created, and in silico modification allows for rapid design of new surrogates.

\subsection{Surrogate Stability Testing}

The serial transfer of the surrogate colonies served as a test of the surrogate's stability in a laboratory environment, and as a test for the recovery of the pEX-A2 plasmid from a culture. While some cells could cure themselves of the plasmid in the absence of ampicillin, both pEX-A2 and pUC19 contain the AmpR gene, so the reintroduction of ampicillin selection would remove untransformed cells from culture.

\subsection{Plasmid Sequencing}

While extraction of a considerable amount of plasmid was possible, the NP surrogate had suboptimal concentrations for sequencing, and because these values were considerably less than what was suggested $(30 \mathrm{ng} / \mu \mathrm{l}$ as opposed to the recommended $100 \mathrm{ng} / \mu \mathrm{l}$ ), two sets of sequencing reactions were performed for each sample (Figure 5). No mismatches occurred, except near the 3' end of the sequencing reaction, where errors in sequencing are more likely. This result indicates that no mutations are likely to have occurred in the surrogate, which is especially important for its role as a positive control, as the point of the surrogate is to replicate a piece of DNA with high fidelity while specifically not translating the sequence into protein.

\subsection{Blood Simulation}

The inoculation of blood samples with the bacterial surrogate, while not perfect, acts as a reasonable facsimile for infected cells in blood. The exact concentration could not be determined, but the cell density was estimated to $10^{6}, 10^{4}$, and $10^{2}$ cells $/ \mathrm{ml}$. As a result, the lowest plasmid concentration was likely between $5.9 \times$ $10^{3}$ and $7.3 \times 10^{3}$ plasmid equivalents $/ \mathrm{ml}$. This is higher than the $1 \times 10^{3}$ virion 
equivalents/ml that is the current bottom limit of detection, and further testing in blood may be required to determine the bottom limit of detection for this surrogate system. However, the concentration of surrogates used in this experiment is within the range that can be seen in an infected blood sample [20]. Because of the rapid doubling rate of $E$. coli, it is problematic to get an exact concentration of bacterial surrogates in a solution. However, the same is true of infected cell samples collected in the field, and it is possible to determine the concentration of plasmid surrogates from purified surrogate stocks, should greater precision be required. As a result, the surrogate system can be used both as a stand-in for infected samples and as a standard for determining concentration. Indeed, the rapid growth rate may be preferable for experiments dealing with detection limits or other detection-over-time protocols.

While pUC and pEX plasmids are suitable for most cloning experiments, they present a few challenges when attempting to create gene surrogates. First, while they have high copy numbers per cell, their specific concentrations are not tightly controlled. As a result, it is difficult to accurately determine the exact amount of plasmid that exists within any single bacterial cell. Second, these plasmids are both double-stranded DNA, which has limited utility for comparison to single-stranded RNA. These plasmids also have a limited maximum insert size, which may become important when working with larger gene segments. Additionally, the pEX-A2 plasmid lacks a functioning lacZ gene, which, by comparison, removes the redundant authentication measure for blue/white selection. The pUC19 plasmid has this selection authentication where inserted surrogate nucleotides interrupt lacZ and prevent the encoded galactosidase from cleaving $\mathrm{x}$-gal into indole, the "blue" in blue/white screening. It is potentially more difficult to authenticate bacteria that have been transformed with the surrogate plasmid pEX-A2 and not unaltered pUC19 plasmid. However, the pEX-A2 plasmid had the EBOV NP surrogate synthesized directly into the plasmid, and was validated through PCR, qPCR and sequencing.

\section{Conclusion}

The results of both the surrogate authentication and detection experiments indicate that the plasmid surrogate had the expected sequence and could be detected with techniques similar to those used to detect the NP gene in EBOV. The insert was found to be oriented correctly, and the sequence was confirmed by plasmid sequencing. The plasmid did not impair the bacterial host, and it did not mutate over a reasonable growth period and 10 replica platings. The observed stability indicates that the surrogate is suitable for work in a typical laboratory setting. For point-of-care diagnostics, the bacterial surrogate's synthetic insert was detected in blood at concentrations as low as $5.8 \times 10^{3}$ plasmid equivalents/ml, which approaches EBOV detection sensitivity $(1 \times$ $10^{3}$ virion equivalents $/ \mathrm{ml}$ ), and the primers designed to be specific to $\mathrm{EBOV}$ were able to bind to the surrogate plasmids. Furthermore, because of the an- 
ti-translational modifications, the bacterial surrogate can be used in a BSL-2 laboratory environment. While it does not mimic EBOV infection and viral replication, the surrogate can serve as a suitable positive control and preliminary test for EBOV NP molecular detection platforms, and the demonstrated techniques could be modified to create reliable surrogates for other pathogens and infectious diseases.

\section{Acknowledgements}

We would like to thank Florida Atlantic University for Assistantship grants to two of the authors DH, BS. Applied Biotech Inc for providing some research reagents and the Three Minute Thesis competition for the opportunity to present this research to our academic community.

\section{Contribution of Authors}

Conceived project (c), directed study (d), dry \& wet lab research (w), contributed to manuscript drafting $(\mathrm{m})$ and manuscript review (r): Esiobu (c, d, $\mathrm{m}, \mathrm{r}$ ), Holmes (w, m, r), Coarsey (w, r), Asghar (r) and Stone ( $m, r)$.

\section{Conflicts of Interest}

The authors declare no conflicts of interest regarding the publication of this paper.

\section{References}

[1] Chippaux, J. (2014) Outbreaks of Ebola Virus Disease in Africa: The Beginnings of a Tragic Saga. Journal of Venomous Animals and Toxins Including Tropical Diseases, 20, 44. https://doi.org/10.1186/1678-9199-20-44

[2] Madelain, V., Nguyen, T.H., Olivo, A., Lamballerie, X.D., Guedj, J., Taburet, A. and Mentré, F. (2016) Ebola Virus Infection: Review of the Pharmacokinetic and Pharmacodynamic Properties of Drugs Considered for Testing in Human Efficacy Trials. Clinical Pharmacokinetics, 55, 907-923. https://doi.org/10.1007/s40262-015-0364-1

[3] Sullivan, N., Yang, Z. and Nabel, G.J. (2003) Ebola Virus Pathogenesis: Implications for Vaccines and Therapies. Journal of Virology, 77, 9733-9737. https://doi.org/10.1128/JVI.77.18.9733-9737.2003

[4] Fischer, R., Judson, S., Miazgowicz, K., Bushmaker, T., Prescott, J. and Munster, V.J. (2015) Ebola Virus Stability on Surfaces and in Fluids in Simulated Outbreak Environments. Emerging Infectious Diseases, 21, 1243-1246. https://doi.org/10.3201/eid2107.150253

[5] Olu, O., Kargbo, B., Kamara, S., Wurie, A.H., Amone, J., Ganda, L., Kasolo, F., et al. (2015) Epidemiology of Ebola Virus Disease Transmission among Health Care Workers in Sierra Leone, May to December 2014: A Retrospective Descriptive Study. BMC Infectious Diseases, 15, Article No. 416. https://doi.org/10.1186/s12879-015-1166-7

[6] Chosewood, L.C. and Wilson, D.E. (2009) Biosafety in Microbiological and Biomedical Laboratories. U.S. Dept. of Health and Human Services, Public Health Ser- 
vice, Centers for Disease Control and Prevention, National Institutes of Health, Washington DC.

[7] Spinato, G., Costantini, G., Fabbris, C., Menegaldo, A., Mularoni, F., Gaudioso, P., Boscolo-Rizzo, P., et al. (2021) The Importance of Early Detection of ENT Symptoms in Mild-to-Moderate COVID-19. Acta Otorhinolaryngologica Italica, 41, 101. https://doi.org/10.14639/0392-100X-N1038

[8] Centers for Disease Control (2015, April 25) Diagnosis. https://www.cdc.gov/vhf/Ebola/diagnosis

[9] Kurosaki, Y., Takada, A., Ebihara, H., Grolla, A., Kamo, N., Feldmann, H., Yasuda, J., et al. (2007) Rapid and Simple Detection of Ebola Virus by Reverse Transcription-Loop-Mediated Isothermal Amplification. Journal of Virological Methods, 141, 78-83. https://doi.org/10.1016/j.jviromet.2006.11.031

[10] Lam, P., Keri, R.A. and Steinmetz, N.F. (2017) A Bioengineered Positive Control for Rapid Detection of the Ebola Virus by Reverse Transcription Loop-Mediated Isothermal Amplification (RT-LAMP). ACS Biomaterials Science \& Engineering, 3, 452-459. https://doi.org/10.1021/acsbiomaterials.6b00769

[11] Coarsey, C., Esiobu, N., Narayanan, R., Pavlovic, M., Shafiee, H. and Asghar, W. (2017) Strategies in Ebola Virus Disease (EVD) Diagnostics at the Point of Care. Critical Reviews in Microbiology, 43, 779-798.

https://doi.org/10.1080/1040841X.2017.1313814

[12] Centers for Disease Control and Prevention (2021, November 8) Ebola Vaccine: Information about ERVEBO ${ }^{\circ}$. Centers for Disease Control and Prevention, Atlanta. https://www.cdc.gov/vhf/ebola/clinicians/vaccine/index.html

[13] Centers for Disease Control and Prevention (2021, February 26) Treatment. Centers for Disease Control and Prevention, Atlanta.

https://www.cdc.gov/vhf/ebola/treatment/index.html

[14] Watt, A., Moukambi, F., Banadyga, L., Groseth, A., Callison, J., Herwig, A., Hoenen, T., et al. (2014) A Novel Life Cycle Modeling System for Ebola Virus Shows a Genome Length-Dependent Role of VP24 in Virus Infectivity. Journal of Virology, 88, 10511-10524. https://doi.org/10.1128/JVI.01272-14

[15] Hoenen, T., Groseth, A., de Kok-Mercado, F., Kuhn, J.H. and Wahl-Jensen, V. (2011) Minigenomes, Transcription and Replication Competent Virus-Like Particles and Beyond: Reverse Genetics Systems for Filoviruses and Other Negative Stranded Hemorrhagic Fever Viruses. Antiviral Research, 91, 195-208. https://doi.org/10.1016/j.antiviral.2011.06.003

[16] Schafer, A.M., Cheng, H., Lee, C., Du, R., Han, J., Perez, J., Rong, L., et al. (2017) Development of Potential Small Molecule Therapeutics for Treatment of Ebola Virus Disease. Current Medicinal Chemistry, 25, 5177-5190. https://doi.org/10.2174/0929867324666171010141416

[17] Przybilla, M., Gandor, C., Mathony, J., Adam, L., Klein, M., Zu, J.U., Jendrusch, M., et al. (2017) iGEM Team Heidelberg 2017-The Phage and the Furious. http://2017.igem.org/Team:Heidelberg

[18] Huang, Y., Xu, L., Sun, Y. and Nabel, G.J. (2002) The Assembly of Ebola Virus Nucleocapsid Requires Virion-Associated Proteins 35 and 24 and Posttranslational Modification of Nucleoprotein. Molecular Cell, 10, 307-316. https://doi.org/10.1016/S1097-2765(02)00588-9

[19] U.S. National Library of Medicine (n.d.) NP Nucleoprotein [Zaire Ebolavirus]Gene-NCBI. National Center for Biotechnology Information, Bethesda. https://www.ncbi.nlm.nih.gov/gene/911830 
[20] Towner, J.S., Rollin, P.E., Bausch, D.G., Sanchez, A., Crary, S.M., Vincent, M., Nichol, S.T., et al. (2004) Rapid Diagnosis of Ebola Hemorrhagic Fever by Reverse Transcription-PCR in an Outbreak Setting and Assessment of Patient Viral Load as a Predictor of Outcome. Journal of Virology, 78, 4330-4341.

https://doi.org/10.1128/JVI.78.8.4330-4341.2004 